\title{
Avaliação da qualidade de xampus anticaspa
}

\section{Evaluation of antidandruff shampoo quality}

Recebido em: 26/06/2018 Aceito em: $18 / 09 / 2018$

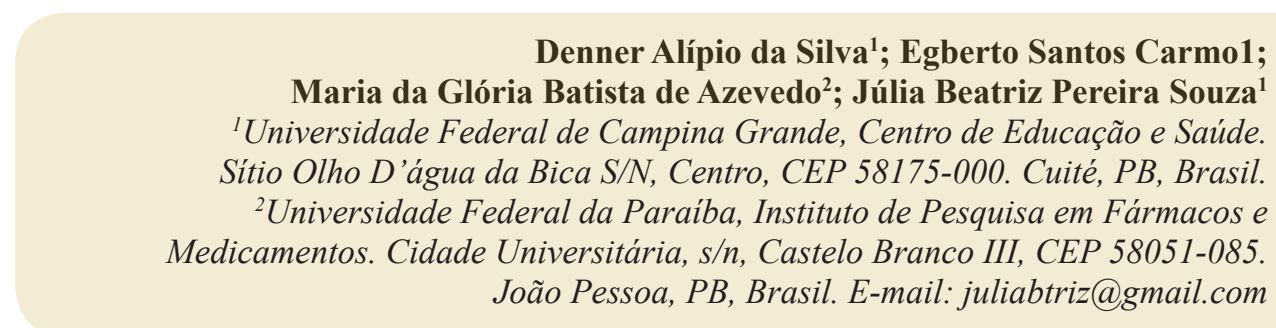

Denner Alípio da Silva'; Egberto Santos Carmo1; Maria da Glória Batista de Azevedo ${ }^{2}$; Júlia Beatriz Pereira Souza ${ }^{1}$ ${ }^{1}$ Universidade Federal de Campina Grande, Centro de Educação e Saúde. Sitio Olho D'água da Bica S/N, Centro, CEP 58175-000. Cuité, PB, Brasil.

${ }^{2}$ Universidade Federal da Paraíba, Instituto de Pesquisa em Fármacos e João Pessoa, PB, Brasil.E-mail: juliabtriz@gmail.com

\section{ABSTRACT}

Superficial mycoses are fungal infections located in various parts of the body, being among the main reasons for dermatological consultations. Among the most common superficial mycoses, dandruff and seborrheic dermatitis are distinguished by their universal distribution and are frequently associated with colonization by fungi of the genus Malassezia. Shampoos containing different antifungal actives such as zinc pyrithione, ketoconazole, and cyclopirox olamine are the main treatments for such conditions. These products, because they have wide use by the population, have the necessity of proving their quality, also verifying their effectiveness, through the quality control. The present study aimed to evaluate the quality parameters of antidandruff shampoos, taking into account the requirements for products for this purpose. Analyzes of the labeled chemical composition, organoleptic, physicochemical characteristics ( $\mathrm{pH}$, viscosity, centrifuge test, density, foam index) and microbiological potency tests were performed, using Candida albicans and Aspergillus niger as biological markers of efficacy. After the tests carried out on five commercial samples, all were approved for organoleptic analysis and centrifugal testing. While about $\mathrm{pH}$, sample 4 was out of standards. In the viscosity test, only sample 5 was performed by that described in the literature. The density values were slightly higher than those found in the literature. However, there is no influence of this parameter on the quality of the evaluated products. In the foam index, samples 1 and 2 showed higher foam consistency. After the efficacy test, only samples 1,2 and 3 were effective in combating the test microorganisms. All samples presented antidandruff actives with proven action in the literature.

Keywords: quality control, dermatomycoses, dandruff.

\section{RESUMO:}

As micoses superficiais são infecções fúngicas localizadas em diversas partes do corpo, estando entre os principais motivos de consultas dermatológicas. Dentre as micoses superficiais mais comuns a caspa e a dermatite seborreica se destacam por terem distribuição universal, sendo frequentemente associadas à colonização por fungos do gênero Malassezia. Xampus contendo diferentes ativos antifúngicos como piritionato de zinco, cetoconazol e ciclopirox olamina, são os principais meios de tratamento para tais afecções. Estes produtos, por terem amplo uso pela população, têm a necessidade de comprovação de sua qualidade, verificando também a sua efetividade, através do controle de qualidade. O presente trabalho teve por objetivo avaliar os parâmetros de qualidade de xampus anticaspa, levando em consideração as exigências para produtos com essa finalidade. Foram realizadas análises da composição química rotulada, das características físico-químicas ( $\mathrm{pH}$, viscosidade, teste de centrífuga, densidade), organolépticas, índice de espuma e ensaios de eficácia microbiológica, utilizando Candida albicans e Aspergillus niger como marcadores biológicos de eficácia. Após a realização dos testes em cinco amostras comerciais, todas foram aprovadas quanto à analise organoléptica e teste de centrifuga. Enquanto que em relação ao pH, a amostra 4 mostrou-se fora dos padrões. 
No teste de viscosidade, apenas a amostra 5 apresentou em conformidade com o descrito na literatura. Os valores de densidade foram ligeiramente maiores que os encontrados na literatura, no entanto, não há influência deste parâmetro na qualidade dos produtos avaliados. No índice de espuma as amostras 1 e 2, apresentaram maior consistência da espuma. Após o ensaio de eficácia, apenas as amostras 1, 2 e 3 mostraram-se eficazes no combate aos microrganismos teste. Todas as amostras apresentaram ativos anticaspa com ação comprovada na literatura.

Palavras-chave: controle da qualidade, dermatomicose, caspa.

\section{INTRODUÇÃO}

As micoses superficiais são infecções fúngicas localizadas nas camadas superficiais da pele e seus anexos, bem como nas mucosas e zonas cutaneomucosas (1). As infecções causadas por fungos estão entre as causas mais comuns de doenças cutâneas. Dentre elas, destacam-se as malassezioses, que são as formas clínicas da infecção causada pela levedura Malassezia. Pitiríase versicolor, foliculite, dermatite seborreica são algumas das doenças associadas ao fungo citado $(2,3)$.

Em relação ao couro cabeludo, a dermatite seborreica e a caspa são alterações crônicas, não contagiosas e recorrentes, em que ocorrem inflamações da pele e couro cabeludo, onde existe um maior número de glândulas sebáceas (4). A caspa, doença que afeta $50 \%$ da população mundial em algum estágio de suas vidas, está relacionada ao fungo lipofílico Malassezia spp., previamente conhecido como Pityrosporum ovale. Em condições normais, estes fungos habitam a superfície do couro cabeludo, vivendo como hóspedes nos seres humanos, retirando o excesso da oleosidade do couro cabeludo, se alimentando dos triglicerídeos (5).

Por se tratar de afecções do couro cabeludo, o xampu é a forma farmacêutica escolhida para um tratamento eficaz destas, no qual podem ser incorporadas várias substâncias antifúngicas, como por exemplo: cetoconazol, piritionato de zinco, óleo de melaleuca, ácido salicílico, enxofre, coaltar, climbazol, piroctona olamina e sulfeto de selênio, sendo esses os principais tratamentos comerciais disponíveis no mercado para estas afecções $(5,6,7)$.

Classificados como produtos de grau de risco 2 (8), os xampus anticaspa precisam que sua qualidade seja controlada por meio de ensaios de referência ou métodos desenvolvidos pela empresa, de forma a garantir a segurança e eficácia para o consumidor. Deve ter comprovada a confiabilidade dos resulta- dos, demonstrando que o procedimento conduz efetivamente ao objetivo desejado (9).

Neste contexto, vários produtos com ação anticaspa e diferentes composições rotuladas são encontrados no mercado, os quais são facilmente acessíveis à população, o que torna a comparação de parâmetros da qualidade importante para comprovar que os produtos realizam de forma eficaz a ação para a qual foram desenvolvidos. Assim, o objetivo do trabalho é avaliar parâmetros da qualidade organoléticos e físico-químicos de diferentes marcas de xampus anticaspa, analisando a composição descrita nos rótulos e a eficácia antifúngica in vitro.

\section{MATERIAL E MÉTODOS}

Amostras. Foram adquiridas cinco amostras de xampus anticaspa, de diferentes marcas de abrangência nacional. Como amostra de referência, utilizou-se um xampu de cetoconazol a 2\% (Ct) manipulado na Farmácia Escola Manoel Casado de Almeida, no Centro de Educação e Saúde. As amostras foram identificadas como 1, 2, 3, 4 e 5, e o padrão manipulado como Ct.

\section{Ensaios Físico-Químicos.}

Viscosidade. A viscosidade das formulações foi avaliada com a utilização de um viscosímetro rotativo analógico, de acordo com o guia de controle da qualidade produtos cosméticos (9). O aparelho foi programado para a escolha de um spindle e rotação a ser testada, em seguida, o spindle foi imerso nas amostras, separadamente e então acionou-se o aparelho. Após a estabilização do aparelho o valor obtido foi utilizado para o cálculo da viscosidade.

pH. O pH foi analisado utilizando um pêagametro digital, por potenciometria, pela determinação da diferença de potencial entre dois eletrodos (o de referência e o de medida) que foram imersos na amostra a ser analisada, após a calibração do apare- 
1ho. O procedimento foi realizado em triplicada. As amostras foram previamente diluídas a $10 \%$ em água destilada (9).

Densidade. A densidade foi determinada utilizando um picnômetro de vidro, de acordo com a metodologia descrita no guia de controle da qualidade de produtos cosméticos (9).

Teste de centrifuga. Foram adicionados $5 \mathrm{~mL}$ de cada amostra em tubos de ensaio específicos para centrifuga, os quais foram submetidos a centrifugação a 3.000 rpm, durante 30 minutos, em temperatura ambiente. A seguir, foram analisados macroscopicamente a fim de avaliar possíveis instabilidades. O teste foi realizado em triplicata e de acordo com o Guia de controle da qualidade de produtos cosméticos (9).

Ensaios organolépticos. As amostras foram avaliadas macroscopicamente quanto às características de cor, odor, aspectos gerais e ausência de qualquer matéria sólida, sujidade, entre outras. Todas deviam apresentar-se adequadas, mantendo-se dentro do padrão de uma amostra líquida (9).

Poder espumante. O poder espumante foi determinado a partir de uma solução aquosa $(0,25 \mathrm{~g}$ da amostra q.s.p. $25 \mathrm{~mL}$ de água) com água destilada, em uma proveta de $100 \mathrm{~mL}$, agitando e girando a coluna 5 vezes. Foi realizada a medida da altura de espuma inicial e após 5 minutos em repouso (10). Ensaio de eficácia antimicrobiana. Para a realização dos testes de comprovação da eficácia antimicrobiana, foi utilizado o método de difusão em ágar. Foi avaliada a capacidade de inibir o crescimento da
Candida albicans e do Aspergillus niger pelas formulações comerciais de xampus anticaspa.

A escolha dos microrganismos para avaliar a capacidade antiproliferativa se justifica pela dificuldade de cultivo da Malassezia e da sensibilidade do cetoconazol, utilizado como padrão de comparação de eficácia, frente ao microrganismo. O Aspergillus niger foi escolhido a fim de verificar a capacidade dos xampus comerciais de inibir microrganismos relacionados com outras dermatomicoses além da caspa.

O ensaio foi realizado utilizando placas de Petri (20 $\mathrm{mm} \times 100 \mathrm{~mm})$ e cilindros de aço inoxidável ( $8 \mathrm{~mm}$ x 6 $\mathrm{mm} \times 10 \mathrm{~mm}$ ). Esse material, assim como a vidraria não volumétrica, utilizados no ensaio microbiológico estavam estéreis.

Preparo do Inóculo. A Candida albicans e o Aspergillus niger foram repicados previamente ao ensaio, 24 e 72 horas antes, respectivamente, em tubo contendo o ágar sabouraud-dextrose (ASD) a $2 \%$ inclinado, mantido à temperatura de $25^{\circ} \mathrm{C}$. Posteriormente, foram preparadas a partir destes microrganismos e solução salina, suspensões a $25 \% \pm 2 \%$ de transmitância a $580 \mathrm{~nm}$. Com estas, foram preparados os inóculos a $0,5 \%$ em ASD a 2 $\%$, mantidos em banho maria a $47^{\circ} \mathrm{C}$ até o momento da distribuição nas placas.

Preparo das soluções padrão e amostras. Foram realizadas diluições para obtenção da concentração teste das amostras e do xampu de cetoconazol utilizado como padrão. A partir das diluições foram obtidas concentrações de $0,5 \%$. As diluições das amostras foram equivalentes à diluição do padrão. 
Quadro 1. Principais componentes químicos dos xampus avaliados

\begin{tabular}{|c|c|c|c|c|c|c|}
\hline \multirow{2}{*}{ Componente } & \multirow{2}{*}{ Função } & \multicolumn{5}{|c|}{ Amostras } \\
\hline & & 1 & 2 & 3 & 4 & 5 \\
\hline Ácido Cítrico & Acidulante & $x$ & & $x$ & $x$ & $x$ \\
\hline Ácido salicílico & Fungicida/Queratolítico & & & & $x$ & \\
\hline Actirox ${ }^{\circledast}$ (Roxitromicina) & Ativo & & & & $x$ & \\
\hline Água & Veículo & $x$ & $x$ & $x$ & $x$ & $x$ \\
\hline Carbomer & Espessante & $x$ & & $x$ & $x$ & \\
\hline Cloreto de Sódio & Doador de viscosidade & $x$ & $x$ & & $x$ & $x$ \\
\hline Coco amido DEA & Tensoativo anfótero & & $x$ & & & $x$ \\
\hline Coco amido propilbetaína & Tensoativo anfótero & $x$ & & $x$ & $x$ & $x$ \\
\hline Destearato glicol & Emoliente & & $x$ & & $x$ & \\
\hline EDTA & Agente quelante & & & & & $x$ \\
\hline Equaderm ${ }^{\circledR}$ & Ativo & & & & $x$ & \\
\hline Hidróxido de Sódio & Conservante & $x$ & & $x$ & $x$ & \\
\hline Lauril Sulfato de Sódio & Tensoativo aniônico & $x$ & $x$ & $x$ & $x$ & $x$ \\
\hline Perfume & Fragrância & $x$ & $x$ & $x$ & $x$ & $x$ \\
\hline Piroctone Olamina & Ativo & & & & $x$ & $x$ \\
\hline Polietileno glicol & Emulsificante & & & & $x$ & \\
\hline Polissorbato 21 & Emulsificante & & & & $x$ & \\
\hline Propileno Glicol & Umectante/conservante & $x$ & & & $x$ & \\
\hline Metilisotiazolinona & Conservante & $x$ & $x$ & $x$ & & $x$ \\
\hline
\end{tabular}

Método de difusão em ágar. Foi feito o cultivo em meio ASD em cada placa de petri. Após a solidificação da camada base, foram vertidos $5 \mathrm{~mL}$ de inóculo (camada semeada com Candida albicans e Aspergillus niger) e após a solidificação foram distribuídos 6 cilindros estéreis em cada placa, nos quais foram adicionados $200 \mu \mathrm{L}$ de cada diluição das amostras e do padrão. Durante o período de análise, as placas foram avaliadas quanto à formação do halo de inibição (11).

Formulação do xampu padrão de cetoconazol 2 \%. O xampu foi formulado com base no Formulário Nacional da Farmacopeia Brasileira (12). Foram preparados $100 \mathrm{~mL}$ do xampu padrão.

\section{RESULTADOS E DISCUSSÃO}

As cinco marcas de xampus analisadas, com diferentes ativos anticaspa, apresentavam diferentes composições químicas, como se poder observado no Quadro 1.
O piritionato de zinco é um agente antimicrobiano de amplo espectro. Possui ação fungistática (contra fungos e leveduras) e bactericida, sendo amplamente utilizado como agente anticaspa e contra a dermatite seborreica, bem como também, na dermatite atópica, psoríase, eczema, micose, pele seca e tinha crural (13, 14). Indicado nas concentrações de 1 a $2 \%$ em xampus, é essencialmente insolúvel em água, portanto sua utilização em xampus é ideal na forma de suspensões (15).

O piroctona olamina, é um fármaco da classe das hidroxipiridonas, que atua como antifúngico bloqueador da reprodução celular. Possui atividade contra leveduras e fungos dermatófitos como a Malassezia spp. e outros fungos filamentosos. É usado em xampus nas concentrações de 0,5 a $1 \%(16,17)$.

O ácido salicílico é utilizado nas formulações de xampus anticaspa atuando no combate à descamação excessiva do couro cabeludo. Por sua ação queratolítica, quando associado com antifúngicos é utilizado no tratamento de infeções fúngicas superficiais, como a caspa e seborreia $(18,19)$. 
Quadro 2. Componentes naturais presentes nos xampus avaliados.

\begin{tabular}{|c|c|c|c|c|c|c|c|}
\hline \multirow{2}{*}{$\begin{array}{l}\text { Componente natural } \\
\text { ou extrato }\end{array}$} & \multirow{2}{*}{ Propriedades } & \multirow{2}{*}{ Referência } & \multicolumn{5}{|c|}{ Amostras } \\
\hline & & & 1 & 2 & 3 & 4 & 5 \\
\hline $\begin{array}{l}\text { Arctium lappa } \\
\text { (Bardana) }\end{array}$ & $\begin{array}{l}\text { Antibacteriana } \\
\text { antifúngica, } \\
\text { fortificante capilar }\end{array}$ & $\begin{array}{l}\text { Holetz et al. (2002); } \\
\text { Messias et al. (2015). }\end{array}$ & & & & & $x$ \\
\hline $\begin{array}{l}\text { Baccharis trimera } \\
\text { (Carqueja) }\end{array}$ & $\begin{array}{l}\text { Antibacteriana e } \\
\text { antifúngica }\end{array}$ & $\begin{array}{c}\text { Avancini, Wiest, Munstock. (2000); } \\
\text { Carreira (2007); } \\
\text { Davicino et al. (2007). }\end{array}$ & & & & & $x$ \\
\hline $\begin{array}{l}\text { Citrus reticulata } \\
\text { (Tangerina); }\end{array}$ & Antifúngica & $\begin{array}{l}\text { Fernandes et al. (2002); } \\
\text { Cavalcanti et al. (2012). }\end{array}$ & & & $x$ & & \\
\hline $\begin{array}{l}\text { Helianthys annuus } \\
\text { (Girassol) }\end{array}$ & Antioxidante & Herbette et al. (2002); & $x$ & & & & \\
\hline Melaleuca alternifolia & $\begin{array}{l}\text { Antisséptica e } \\
\text { antifúngica }\end{array}$ & $\begin{array}{c}\text { Oliveira, Bloise (1999); } \\
\text { Batistuzzo, Itaya, Eto. (2002); } \\
\text { Packer et al. (2007). }\end{array}$ & $x$ & $x$ & $x$ & & \\
\hline $\begin{array}{c}\text { Menta piperita } \\
\text { (Menta pimentinha) }\end{array}$ & $\begin{array}{l}\text { Antibacteriana e } \\
\text { antifúngica }\end{array}$ & $\begin{array}{l}\text { De Matos et al. (2009); } \\
\text { Michelin et al. (2005) }\end{array}$ & $x$ & & & & \\
\hline Própolis & $\begin{array}{l}\text { Antibacteriano e } \\
\text { antifúngico }\end{array}$ & $\begin{array}{l}\text { Packer et al. (2007); } \\
\text { Molina et al. (2010); } \\
\text { Pinto et al. (2004) }\end{array}$ & & & & & $x$ \\
\hline $\begin{array}{l}\text { Prunus amygdalus dulcis } \\
\text { (amêndoas) }\end{array}$ & $\begin{array}{l}\text { Condicionante e } \\
\text { umectante }\end{array}$ & Cabrera Macas, Tene, Elizabeth (2013) & & $x$ & & & \\
\hline Citrus aurantium & Emoliente & Grollier, et al. (1990) & & & & & \\
\hline
\end{tabular}

Além desses, outros ativos anticaspa são utilizados, como a roxitromicina (Actirox ${ }^{\circledR}$ ), um macrolídeo semissintético derivado e com aspecto semelhante a eritromicina (20). Não foram encontradas na literatura informações sobre o princípio ativo Equaderm ${ }^{\circledR}$, pois trata-se de uma substância patenteada pela fabricante do xampu. Além dos já citados no quadro 1 , alguns componentes naturais foram observados nos rótulos das amostras testadas, os quais estão descritos no quadro 2. Apenas a formulação 4 não apresentava extrato vegetal ou algum outro componente natural em sua composição. Nas formulações de xampus são utilizadas combinações com extratos vegetais a fim de se conseguir fórmulas alternativas, com perfil eficaz e baixa agressividade que possam ser utilizadas por uma vasta gama de indivíduos (13).

Entre os extratos vegetais encontrados destaca-se a própolis, utilizada como auxiliar no combate a caspa, pois tem seu efeito na atividade extracelular da fosfolipase (inibindo a enzima) e adesão fúngica nas células epiteliais (21) e o óleo de melaleuca (Melaleuca alternifolia) que é um dos componentes naturais ativos mais utilizados nos xampus anticaspa, obtido das folhas e ramos terminais da planta. Possui ação antifúngica e antisséptica comprovada na prática e in vitro, não sendo tóxica ou corrosiva para os tecidos (16).

O controle da qualidade de medicamentos e cosméticos deve ser realizado de forma a garantir a eficácia, qualidade e segurança destes produtos, bem como, garantir a aceitabilidade do produto pelos usuários, analisando de forma concisa propriedades organolépticas, físicas e químicas dos mesmos.

A avaliação organoléptica fornece parâmetros que objetivam avaliar, de imediato, o estado em que se encontra a amostra em estudo por meio de análises comparativas, verificando alterações como: separação de fases, precipitação e turvação, permitindo o reconhecimento primário do produto, determinando assim os parâmetros de aceitação do produto pelo consumidor (9). Todas as marcas avaliadas se mostraram dentro dos padrões, não apresentando nenhum tipo de alteração, conforme informações do Quadro 3. Por não existir regulamentação que padronize a fabricação dos xampus, cada marca pode atribuir o aspecto que desejar à sua formulação, utilizando-se da estética do produto como uma característica relevante na escolha e aceitação do mesmo, desde que não represente problemas relacionados à segurança e eficácia do produto (22). A Figura 1 mostra da diversidade de aspecto visual entre as amostras. 
Quadro 3. Avaliação das características organolépticas dos xampus avaliados

\begin{tabular}{|c|c|c|c|}
\hline Amostra & Cor & Odor & Aspecto \\
\hline 1 & Branco & Característico & Cremoso/brilhoso \\
\hline 2 & Branco & Característico & Cremoso/brilhoso \\
\hline 3 & Azulado & Característico & Cremoso/Perolado \\
\hline 4 & Esverdeado & Característico & Cremoso/Perolado \\
\hline 5 & Amarelado & Característico & Fluido/transparente \\
\hline
\end{tabular}

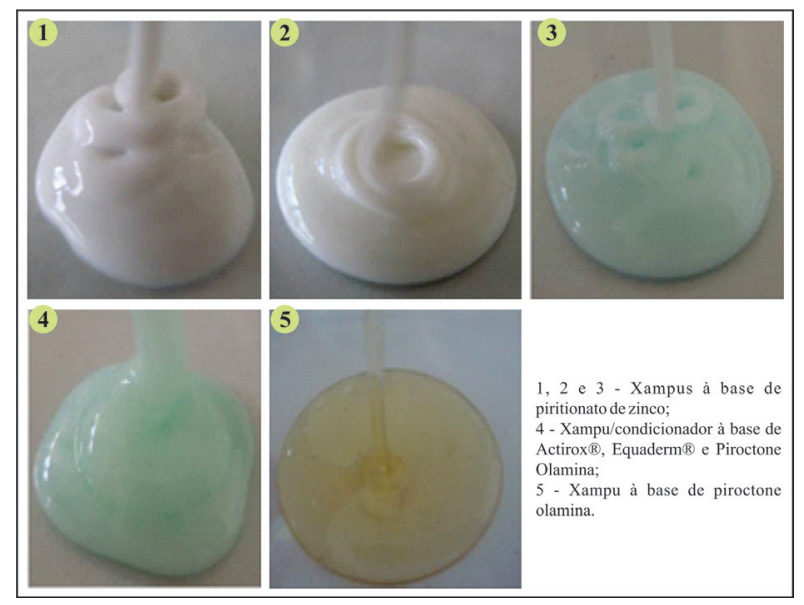

Figura 1. Aspecto visual das amostras de xampus anticaspa analisados

De acordo com a Tabela 1, o pH dos xampus avaliados variou de 4,93 a 7,05. Dessa forma, eram compatíveis com o $\mathrm{pH}$ fisiológico do couro cabeludo e próximos da faixa ideal para a saúde do fio capilar.

Tabela 1. Parâmetros físicos químicos dos xampus avaliados

\begin{tabular}{cccc|} 
Amostra & $\begin{array}{c}\text { Viscosidade } \\
(\mathbf{c P})\end{array}$ & $\begin{array}{c}\text { Densidade } \\
\left(\mathbf{g} / \mathbf{c m}^{\mathbf{3}}\right)\end{array}$ & $\mathbf{p H}$ \\
\hline 1 & 9333,33 & 1,07 & 6,79 \\
\hline 2 & 9833,33 & 1,08 & 7,05 \\
\hline 3 & 10166,66 & 1,06 & 6,95 \\
\hline 4 & 11750,00 & 1,05 & 4,93 \\
5 & 2166,66 & 1,03 & 7,0 \\
\hline
\end{tabular}

O pH dos xampus, de modo geral, está próximo da neutralidade, porém é preferível que apresente valores de $\mathrm{pH}$ compatíveis com o $\mathrm{pH}$ da pele, ligeiramente baixo (entre 5,0 e 7,0). Entretanto, um pH menor que 5,0 não chega a ser um grande problema, tendo em vista que o $\mathrm{pH}$ da pele está em torno de 5,5 e do couro cabeludo entre 4,2 e 5,6. No caso da existência de algum distúrbio que altere o $\mathrm{pH}$ do couro cabeludo, como na dermatite seborreica, o xampu irá regularizar mantendo sua acidez normal $(23,24)$.
É importante ressaltar que o couro cabeludo sofre agressão física com o surgimento da caspa. Assim, caso o pH não seja o ideal, podem ocorrer outros problemas como inflamação, levando desconforto ao usuário, o que muitas vezes desencadeia a interrupção do uso do xampu (13).

Quanto ao parâmetro viscosidade (Tabela 1), os valores variaram de 2166 a $11750 \mathrm{cP}$. A amostra 5 apresentou viscosidade bem inferior às demais, mas dentro dos padrões comerciais, uma vez que, grande parte dos xampus comercializados apresenta viscosidade entre 2000 e 5000 cP conforme observado por Oliveira e cols (2013) (13).

De acordo com Cunha e cols. (2009), os xampus anticaspa, devem apresentar uma boa viscosidade, permitindo uma maior aderência do produto ao couro cabeludo, o que irá facilitar a ação antimicrobiana (25). Porém, a viscosidade deve permitir o escoamento da embalagem, o que pode ser prejudicado por valores muito altos. No entanto, embora as amostras 1, 2, 3 e 4 tenham apresentado viscosidade bem superior a $5000 \mathrm{cP}$, não houve quaisquer problemas de escoamento durante a retirada da embalagem.

O teste de centrífuga permite a observação e constatação de alterações possíveis na estrutura da formulação (9). Na avaliação das cinco marcas de xampu, não foi observada qualquer alteração, como separação de fases, caking ou precipitação, sem indícios de instabilidades que pudessem comprometer a qualidade do produto.

Quanto à densidade, os valores encontrados variaram de 1,03 a $1,08 \mathrm{~g} / \mathrm{cm}^{3}$. De acordo com Ferreira (2010), de forma geral, a densidade dos xampus e sabonetes líquidos encontra-se entre 1,010 e $1,020 \mathrm{~g} / \mathrm{cm}^{3}$ (23). Lourenço e Lyra (2014), ao avaliar a estabilidade de xampus anticaspa à base de piritionato de zinco $2 \%$, observaram densidades relativas entre 1,01 a $1,04 \mathrm{~g} / \mathrm{cm}^{3}$ (14). Apesar dos valores aferidos neste trabalho serem ligeiramente superiores que os encontrados na literatura, 
não há nenhuma influência deste parâmetro na qualidade e eficácia dos produtos testados.

Com relação ao índice de espuma (Tabela 2), a altura das colunas formadas variou de $4,0 \mathrm{~cm}$ a $7,1 \mathrm{~cm}$. Três das amostras $(3,4$ e 5) apresentaram decaimento da espuma após o tempo de 5 minutos.

Tabela 2. Índice de espuma dos xampus avaliados

\begin{tabular}{|c|c|c|c|c|c|}
\hline & \multicolumn{5}{c|}{ Amostras } \\
\hline Tempo & 1 & 2 & 3 & 4 & 5 \\
\hline $0^{\prime}$ & $4,8 \mathrm{~cm}$ & $5,6 \mathrm{~cm}$ & $7,1 \mathrm{~cm}$ & $4,3 \mathrm{~cm}$ & $4,0 \mathrm{~cm}$ \\
\hline $5^{\prime}$ & Estável & Estável & $6,9 \mathrm{~cm}$ & $4,0 \mathrm{~cm}$ & $3,5 \mathrm{~cm}$ \\
\hline
\end{tabular}

Mesmo não apresentando os maiores níveis, a espuma formada pelas amostras 1 e 2 mostrou-se mais consistente que as demais, que eram menos densas e com decaimento mais rápido ao longo do tempo. A capacidade espumante de uma formulação é um parâmetro importante, pois o consumidor compara sua eficácia de limpeza com a quantidade, textura e persistência da espuma formada durante o processo de uso, de modo a julgar a qualidade e eficácia do produto pela sua capacidade geradora de espuma; dessa forma, para o consumidor, se analisada apenas esta variável, a amostra 3 seria julgada como sendo a de melhor qualidade, pois apresentou uma maior quantidade de espuma com pouco decaimento (26).
Para os ensaios de eficácia antimicrobiana, devido à dificuldade de cultivo da Malassezia, a escolha da $C$. albicans como microrganismo revelador fundamentou-se nos requisitos básicos para a escolha de um organismo para os testes de potência antimicrobiana como ensaio do controle de qualidade, que são: a sensibilidade à substância em análise e a capacidade de crescimento rápido, e, se possível, que não seja patogênico (27).

Um estudo de caso conduzido por Chokoeva e cols. (2016) mostra a incidência de dois casos distintos de Tinea capitis ocasionado por um fungo filamentoso, o Aspergillus niger (28). Os autores sugeriram esse fungo como sendo um novo agente etiopatogênico da Tinea capitis, além dos fungos dermatófitos. Dessa forma, o Aspergillus niger, foi escolhido como um segundo microrganismo teste para o experimento, verificando assim, a capacidade dos xampus comerciais utilizados no tratamento anticaspa, de inibir o crescimento de outros fungos envolvidos com outras dermatomicoses.

De acordo com a Tabela 3, pode-se observar que as amostra $1 \mathrm{e} 2$, à base de piritionato de zinco, tiveram uma maior atividade contra as cepas de Aspergillus niger e Candida albicans, respectivamente, e consequentemente uma maior atividade antimicrobiana em relação às demais amostras, formando um halo maior.

Tabela 3. Halos de inibição em mm das amostras analisadas nas diluições recomendadas de uso sobre os microrganismos testes pelo método de difusão em ágar $(\mathrm{n}=5)$

\begin{tabular}{|c|c|c|c|c|}
\hline \multirow{2}{*}{ Amostra } & \multicolumn{4}{|c|}{ Microrganismo } \\
\cline { 2 - 5 } & \multicolumn{2}{|c|}{ Candida albicans } & Média & CV (\%) \\
\cline { 2 - 5 } & Média & CV $(\%)$ & - & - \\
\hline Cetoconazol $(200 \mu \mathrm{g} / \mathrm{mL})$ & 15,8 & 6,93 & 15,20 & 2,94 \\
\hline 1 & 23,60 & 4,83 & 14,60 & 6,13 \\
\hline 2 & 23,80 & 5,48 & 11,80 & 7,09 \\
\hline 3 & 19,00 & 5,26 & - & - \\
\hline 4 & - & - & - & - \\
\hline
\end{tabular}

Legenda: (-): Não houve formação do halo de inibição.

O antimicrobiano se difunde no ágar em concentrações decrescentes, enquanto que a cepa semeada mantém seu crescimento até encontrar a concentração inibitória mínima. A partir do ponto de aplicação, ocorre a formação de um halo de inibição ao redor do cilindro (29). O halo é determinado em milímetros e é direta- mente proporcional à potência do antimicrobiano, assim, halos maiores indicam maior atividade e eficácia do antimicrobiano. Porém, o aumento do diâmetro do halo ocorre até que se esgote a capacidade de difusão do antimicrobiano no ágar (29). Os halos de inibição formados podem ser visualizados na Figura 2. 


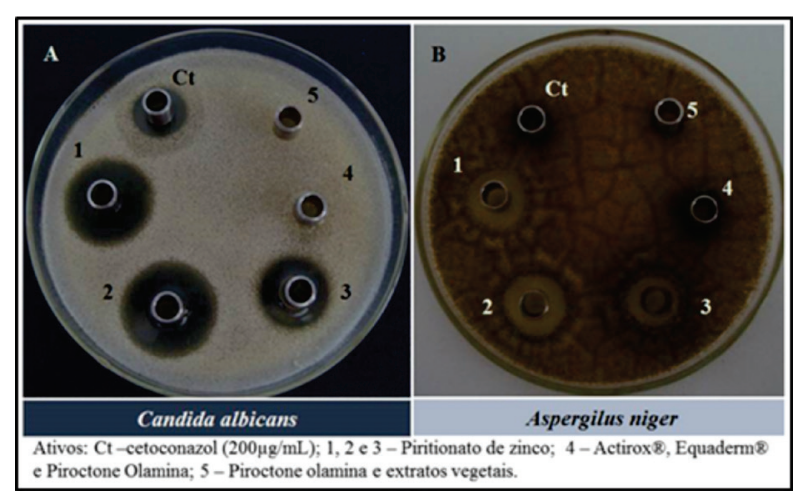

Figura 2. Atividade antimicrobiana dos xampus anticaspa sobre os microrganismos teste pelo método de difusão em ágar

Observando a Tabela 3 e a Figura 2, percebe-se a não formação do halo de inibição do crescimento do Aspergillus niger com ct, subtendendo-se que o cetoconazol, utilizado como padrão, não foi capaz de inibir o crescimento do microrganismo, tendo-se como justificativa uma possível resistência da cepa utilizada no experimento ao cetoconazol. Por outro lado, houve inibição do crescimento do microrganismo nas amostras à base de piritionato de zinco, sendo que as amostras 1 e 2 apresentaram diâmetro maior do halo formado, indicando uma maior atividade antifúngica contra o microrganismo. Não ocorreu formação de halos de inibição nas demais amostras, a base de piroctona olamina e demais ativos anticaspa.

Após os cálculos do coeficiente de variação, pode ser verificado que os valores variaram de 4,83 a 7,09\%, dentro dos limites aceitáveis de confiabilidade dos ensaios realizados, apresentando precisão adequada para o doseamento microbiológico para avaliar a eficácia antimicrobiana dos xampus anticaspa frente às cepas de Candida albicans e Aspergillus niger. De acordo com o guia para validação de métodos analíticos e bioanalíticos (RE 899/03) da Anvisa, o valor máximo aceitável do coeficiente de variação deve ser definido de acordo com a metodologia aplicada, a concentração do analito na amostra, o tipo de matriz e a finalidade do método, não sendo admitidos valores superiores a $15 \%$ para métodos biológicos (30).

As classes de medicamentos mais utilizados em infecções por Aspergillus e que mostram ação in vitro contra grande variedade de espécies desse gênero são os polienos, como anfotericina $\mathrm{B}$, e os derivados imidazólicos, como cetoconazol (31-33). Entretanto, a resistência de espécies de Aspergillus a alguns antifúngicos usados na clínica tem sido a causa de prognóstico clínico preocupante (34-36). Por vários anos, os antimicrobianos têm sido usados indiscriminadamente para o tratamento de infecções resultando no aparecimento de cepas resistentes $(37,38)$.

O cetoconazol, considerado como um agente antifúngico de amplo espectro possui atividade contra infecções clínicas de Candida spp. $(19,39)$. O cetoconazol prejudica a biossíntese do ergosterol na membrana citoplasmática e conduz ao acúmulo de 14- $\alpha$-metilesterois. Esses metilesterois podem desagregar o arranjo compacto das cadeias acíclicas dos fosfolipídios e prejudicar as funções de determinados sistemas enzimáticos ligados à membrana, como a ATPase e enzimas do sistema do transporte de elétrons, inibindo, consequentemente, o crescimento dos fungos (40).

Um estudo comparativo desenvolvido por Pierard-Franchimont e cols. (2002) mostrou que o cetoconazol é mais efetivo que o piritionato de zinco no tratamento e controle da seborreia e da caspa, sendo a melhor opção para o tratamento dessas afecções reduzindo os níveis de descamação no couro cabeludo em quantidade superior ao piritionato de zinco $(6,41)$.

As amostras 1, 2 e 3, além de conterem o piritionato de zinco como ativo anticaspa em sua composição, possuiam em comum o óleo de Melaleuca alternifolia que possui atividade antifúngica e antisséptica comprovada, sendo eficaz no tratamento da caspa, justificando assim a melhor atividade destas amostras. As amostras contendo piroctona olamina (4 e 5) não impediram o crescimento dos microrganismos, não apresentando formação de halo de inibição. Contudo, testes de suscetibilidade in vitro contra Candida spp, mostraram melhor atividade antifúngica de piroctona olamina comparada a fármacos convencionais (42).

Desta forma, a não formação de halos pelas amostras 4 e 5 , indica que os ativos presentes nas distintas formulações não foram eficazes nas condições experimentais utilizadas, com ambos os bioindicadores (Candida e Aspergillus). Isso pode ser um indicativo de problemas como concentração inadequada de constituinte ativo, interações entre os componentes da formulação inibindo a atividade, e até mesmo ineficácia da substância, que pode estar relacionada a baixa qualidade da matéria-prima utilizada, cabendo estudos adicionais para melhor investigação das formulações ou dos ativos em questão.

Um xampu anticaspa eficiente precisa fornecer um alívio dos sintomas para garantir a adesão do paciente, como também fornecer benefícios cosméticos, pois trata-se de um produto de higiene pessoal com grau de risco 2, cuja formulação deve cumprir com a definição ado- 
tada e possui indicação específica, cujas características exigem comprovação de segurança e/ou eficácia, bem como informações e cuidados, modo e restrições de uso. As diferentes performances observadas nos parâmetros de qualidade avaliados neste estudo apontam para o controle da qualidade como uma importante ferramenta que se destina a proteger a população, cujo objetivo maior é a garantia da qualidade dos produtos a serem consumidos.

\section{CONCLUSÃO}

A avaliação da composição dos rótulos permitiu observar que os xampus possuem diferentes ativos (piritionato de zinco, piroctona olamina, Actirox ${ }^{\circledR}+$

\section{REFERÊNCIAS}

1. Somenzi CC, Ribeiro TS, Menezes A. Características Particulares da Micologia Clínica e o Diagnóstico Laboratorial de Micoses Superficiais. NewsLab, 2006;77:106118.

2. Staub I, Cruz AS, Pinto TJA, Schapoval EES, Bergold AM. Determinação da segurança biológica do xampu de cetoconazol: Teste de irritação ocular e avaliação do potencial de citotoxicidade in vitro. Rev. Bras. Cienc. Farm., 2007;43(2):301-307. DOI: 10.1590/S151693322007000200017

3. Azevedo MGB. Estudo da solubilização do cetoconazol por microemulsão para incorporação em xampu. [Trabalho de conclusão de curso]. Cuité: Centro de Educação e Saúde, Universidade Federal de Campina Grande, 2012.

4. Steiner D. Dermatite seborreica. Cosmetics \& Toiletres, 1998;10(1):26.

5. Berti J, Rodrigues J, Lodo C, Leonardi GR. Substâncias ativas utilizadas em produtos anticaspa. Infarma, 2007;19(9/10):29-32.

6. Staub I. Avaliação da fotoestabilidade do cetoconazol e determinação da atividade antifúngica e da segurança biológica in vivo e in vitro do xampu de cetoconazol. [Tese]. Porto Alegre: Programa de Pós-graduação em Ciências Farmacêuticas, Universidade Federal do Rio Grande do Sul, 2005.

7. Grimalt R. A practical guide to scalp disorders. Journal of Investigative Dermatology Symposium Proceedings, 2007;12(2):10-14. DOI: 10.1038/sj.jidsymp.5650048

8. BRASIL. Agência Nacional de Vigilância Sanitária. RDC $n^{\circ} 7$, de 10 de fevereiro de 2015. Dispõe sobre os requisites técnicos para regularização de produtos de higiene pessoal, cosméticos e perfumes. Diário Oficial da União, $n^{0}$ 29, 11 de fevereiro de 2015. Seção 1. p. $39-42$.
Equaderm $\left.{ }^{\circledR}\right)$ cujas ações anticaspa são relatadas na literatura.

Quanto às características organolépticas e físico-químicas, todas as amostras estavam dentro dos padrões aceitáveis de qualidade; somente o parâmetro viscosidade mostrou grande variação entre as amostras, mas sem problemas de escoamento que comprometesse o uso dos produtos. O índice de espuma mostrou maior consistência e durabilidade para as amostras 1 e 2 .

Quanto à eficácia, apenas as amostras à base de piritionato de zinco e Melaleuca alternifolia (1, 2 e 3) mostraram-se efetivas no combate aos microrganismos testes, apresentando melhor atividade quando comparadas ao padrão de referência
9. BRASIL. Agência Nacional de Vigilância Sanitária. Guia controle de qualidade de produtos cosméticos. 2.ed. Brasília: Anvisa. 2008.

10. Isaac VLB, Cefali LC, Chiari BG, Oliveira CCLG, Salgado HRN, Corrêa MA. Protocolo para ensaios físico-químicos de estabilidade de fitocosméticos. Rev Ciênc Farm Básica Apl. 2008;29(1):81-96.

11. BRASIL. Agência Nacional de Vigilância Sanitária. Farmacopeia Brasileira, volume 1. 5.ed. Brasília: Anvisa, 2010.

12. BRASIL. Agência Nacional de Vigilância Sanitária. Formulário Nacional da Farmacopeia Brasileira. $2^{\circ}$ ed. Brasília: Anvisa, 2012.

13. Oliveira MA, Faria MB, Andrade WM, Fernandes CKC. Avaliação da estabilidade e atividade antifúngica de formulações de xampu anticaspa contendo piritionato de zinco e a influência da adição de extratos vegetais. Revista eletrônica FMB, 2013;6(1):1-21.

14. Lourenço EAD, Lyra MAMM. Desenvolvimento e estudo de estabilidade de Xampu Anticaspa a base de Piritionato de Zinco 2\%. Revista eletrônica da Estácio Recife, 2015;1(1): 2015.

15. Martindale: The Complete Drug Reference. 36. ed. London: Pharmaceutical Press; 2009.

16. Batistuzzo JAO, Itaya M, Eto Y. Formulário Medico-Farmacêutico. 4. ed. São Paulo: Pharmabooks Editora, 2011

17. Magagnin CM. Caracterização fenotípica e genotípica de isolados clínicos de trichophyton sp. do estado do Rio Grande do Sul. [Dissertação]. Porto Alegre: Programa de Pós-Graduação em Medicina: Ciências Médicas, Universidade Federal do Rio Grande do Sul, 2013.

18. Garcia BGBC, Stahlke ERS, Vieira IR, Callegari IC, Caldas LSC, Mendes PHO, et al. Cosmiatria: manual dermatológico farmacêutico. Guarapuava (PR): Grafel; 2006. 
19. Korolkovas A, França FFAC. Dicionário Terapêutico Guanabara. Ed. 2005/2006. Rio de Janeiro: Guanabara Koogan, 2006.

20. Auto HF, Constant JMC, Costant ABL. Antibióticos e Quimioterápicos. 5. Ed. Maceió: Editora Edufal, 2008.

21. D'auria FD, Tecca M, Scazzocchio F, Renzini V, Strippoli V. Effect of Propolis on Virulence Factors of Candida albicans. J. Chemother, 2013;15(5):454-460. DOI: 10.1179/joc.2003.15.5.454

22. Bezerra PX, SOUZA JBP, Carmo ES. Avaliação da Rotulagem e Parâmetros de Qualidade de Sabonetes Íntimos. Rev Bras Ciências da Saúde, 2016;20(1:51-60. DOI: 10.4034/RBCS.2016.20.01.08

23. Ferreira AO. Guia prático da farmácia magistral. 4. ed. São Paulo: Pharmabooks Editora, 2010.

24. Lima GCG, Comarella LC. Sugestão de desenvolvimento de formulações de xampu-sabonete auxiliares no tratamento da dermatite seborreica. Rev Uniandrade, 2013;13(2):160-174.

25. Cunha AR, Silva RS, Chorilli M. Desenvolvimento e avaliação da estabilidade física de formulações de xampu anticaspa acrescidas ou não de extratos aquosos de hipérico, funcho e gengibre. Rev. Bras. Farm, 2009;90(3):190-195.

26. Almeida MAA, Azevedo MGB, Falcão JSA. Avaliação da estabilidade preliminar do extrato aquoso de neem (azadirachta indica) em xampu. Educação, Ciência e Saúde, 2014;1(1):31-45. DOI: 10.20438/ecs.v1i1.5

27. Baird RM., Hodges NA., Denyer S. Handbook of microbiological quality control in pharmaceuticals and medical devices. 1. Ed. CRC Press, 2000.

28. Chokoeva AA, Zisova L, Chorleva K, Tchernevc G. Aspergillus niger - a possible new etiopathogenic agent in Tinea capitis? Presentation of two cases. Braz J Infect Dis, 2016;20(3):303-307. DOI: 10.1016/j.bjid.2016.01.002

29. Esmerino LA, Pereira AV, Adamowicz T, Borges DM, Talacimon EA, Schelesky ME. Método microbiológico para determinação da potência de antimicrobianos. Publ. UEPG Cienc Biol Saúde, 2004;10(1):53-60. DOI: 10.5212/publicatio\%20uepg.v10i1.382

30. BRASIL. Agência Nacional de Vigilância Sanitária. RE 899 , de 29 de maio de 2003. Guia para validação de métodos analíticos e bioanalíticos. Diário Oficial da União $n^{\circ} 104$, de 02 de junho de 2003. Seção 1. p. 56-59.
31. Brody TM, Larner J, Minneman KP, Neu HC. Farmacologia humana: da molecular à clínica. $2^{\mathrm{a}}$ ed. Rio de Janeiro (RJ): Guanabara Koogan; 1997.

32. Oliveira PR, Resende SM, Oliveira FC, Oliveira AC. Ceratite fúngica. Arq Bras Oftalmol. 2001;64(1):75-9. DOI: 10.1590/S0004-27492001000100015

33. Hawkins EC. Distúrbios do sistema respiratório. Medicina interna de pequenos animais. 4. ed. Rio de Janeiro: Elsevier, 2010.

34. Moore CB, Sayers N, Mosquera J, Slaven J, Denning DW. Antifungal drug resistance in Aspergillus. J. Infection, 2000;41(3):203-220. DOI: 10.1053/jinf.2000.0747

35. Canuto MM, Rodero FG. Antifungal drug resistance to azoles and polyenes. The Lancet infectious diseases, 2002;2(9):550-563. DOI: 10.1016/S14733099(02)00371-7

36. Curtis L, Cali S, Conroy L, Baker K, Ou CH, Hershow R, Norlock-Cruz F, Scheff P. Aspergillus surveillance project at a large tertiary-care hospital. J Hospital Infection, 2005;59(3):188-196. DOI: 10.1016/j.jhin.2004.05.017

37. Desselberger, U. Emerging and re-emerging infectious disease. J Infection, 2000;40(1):3-15. DOI: 10.1053/ jinf.1999.0624

38. Kontoyiannis DP, Lewis RE. Antifungal drug resistance of pathogenic fungi. The Lancet, 2002;359(9312):11351144. DOI: 10.1016/S0140-6736(02)08162-X

39. Pons Júnior FR. Suspensões e formulações tópicas contendo nanocápsulas e micropartículas de cetoconazol: avaliação da estabilidade e atividade antimicrobiana. [Dissertação]. Santa Maria: Centro Universitário Franciscano, 2011.

40. Hardman JG, Limbird LE. As bases farmacológicas da terapêutica. 10. ed., McGraw-Hill: Rio de Janeiro, 2003.

41. Pierard-Franchimont C, Goffin V, Decroix J, Pierard GE. A multicenter randomized trial of ketoconazole $2 \%$ and zinc pyrithione $1 \%$ shampoos in severe dandruff and seborrheic dermatitis. Skin Pharmacol Appl Skin Physiol. 2002;15:434-441. DOI: 10.1159/000066452

42. Couto FMMD. Fungemia e ação antifúngica e antitumoral de isoflavonas da soja e hidroxipiridonas [Tese]. Recife: Universidade Federal de Pernanbuco, 2013. 